
\title{
28 Research Suare \\ Prehospital Triage Tools across the World: A Scoping Review of the Published Literature
}

\section{Smitha Bhaumik}

Denver Health and Hospital Authority

\section{Merhej Hannun}

St George's Hospital Medical School: St George's University of London

\section{Chelsea Dymond}

Denver Health and Hospital Authority

\section{Kristen DeSanto}

University of Colorado Denver School of Medicine

\section{Whitney Barrett}

Denver Health and Hospital Authority

\section{Lee A Wallis}

University of Cape Town

Nee-Kofi Mould-Millman ( $\nabla$ nee-kofi.mould-millman@ucdenver.edu )

University of Colorado, School of Medicine https://orcid.org/0000-0003-4303-6903

\section{Research article}

Keywords: prehospital, EMS, emergency medical services, triage, scoping review, global health, international, trauma, stroke

Posted Date: January 27th, 2021

DOl: https://doi.org/10.21203/rs.3.rs-153478/v1

License: (c) (1) This work is licensed under a Creative Commons Attribution 4.0 International License. Read Full License 


\section{Abstract}

\section{Background}

Accurate triage of the undifferentiated patient is a critical task in prehospital emergency care. This scoping review aims to identify published tools used for prehospital triage across the world and describe their performance characteristics.

\section{Methods}

A comprehensive search was performed of primary literature in English-language journals from 2009 to 2019. Papers included focused on emergency medical services (EMS) triage of single patients. Two blinded reviewers and a third adjudicator performed independent title and abstract screening and subsequent full-text reviews.

\section{Results}

Of 1521 unique articles, 55 (3.6\%) were included in the final synthesis. The majority of prehospital triage tools focused on stroke $(n=19 ; 35 \%)$, trauma $(19 ; 35 \%)$, and general undifferentiated patients $(15 ; 27 \%)$. All studies, and resulting articles, were performed in high income countries, with the majority in North America $(23,42 \%)$ and Europe $(22,40 \%) .4(7 \%)$ articles focused on the pediatric population. The general triage tools aggregated prehospital vital signs, mental status assessments, and sometimes, features of the history, exam, and anticipated resource need, to categorize patients into numerical or color categories to represent level of acuity. The studies assessed the tools' ability to accurately predict emergency department triage assignment, hospitalization and short-term mortality. The stroke triage tools were designed to promote rapid identification of patients with acute large vessel occlusion ischemic stroke to trigger timely transport to diagnostically- and therapeutically-capable hospitals. Stroke triage literature evaluates tools' diagnostic performance, impact on tissue plasminogen activator administration rates, and correlation with in-hospital stroke scales. Trauma triage tools sought to identify patients that require immediate transport to trauma centers with emergency surgery capability. The studies on trauma triage evaluate prediction of trauma center need, under-triage and over-triage rates for major trauma, and survival to discharge.

\section{Conclusions}

The published literature on prehospital triage tools predominantly derive from high-income health systems and mostly focus on adult stroke and trauma populations. Most studies sought to further simplify existing triage tools, without sacrificing triage accuracy, or assessed the predictive capability of the triage tool. There was no clear 'gold-standard' singular prehospital triage tool for acute undifferentiated patients.

Trial Registration 
Not applicable.

\section{Background}

Emergency medical services (EMS) systems deliver prehospital care and transport to victims of sudden illness or injury [1]. A critical task in prehospital emergency care is the accurate triage of the undifferentiated patient which helps dictate the ensuing treatment and/or transportation plan. Triage has a demonstrated mortality benefit, for example, in the setting of ST-segment elevation myocardial infarction (STEMI), stroke and trauma [2,3,4]. Triage is employed repeatedly and used across the spectrum of emergency care delivery: at the time of resource dispatch, upon prehospital personnel arrival at the scene of the patient, and again by staff of the receiving facility [5].

In low- and middle-income countries (LMICs) in particular, prehospital triage may play an even more critical role. It is estimated that more than half of deaths in LMICs are caused by conditions that benefit from prehospital and emergency care. Examples include infectious diseases, complications of pregnancy, cardiovascular disease, road traffic accidents and interpersonal violence [6]. Many LMICs have nascent EMS systems that would benefit from effective triage tools $[7,8,9]$.

Regardless of country or setting, there is a general paucity of review literature appraising prehospital triage tools currently in use across the world. The only comprehensive search of prehospital triage tools to date is a 2013 systematic review which assessed patient-level outcomes attributable to validated prehospital triage systems [10]. Despite screening over 11,000 unique titles and abstracts, and performing 120 full text reviews, the authors found no studies that met their inclusion criteria, which required the triage tool to undergo direct comparison to an alternate tool or to a no triage arm. Hence, more studies are needed to better understand the state of prehospital triage across the globe in an effort to inform future EMS research and development.

The primary aim of this scoping review was to identify the breadth and diversity of published prehospital triage tools in use across the world and to understand reasons why these studies were performed. Secondarily, we sought to describe the performance characteristics of these tools to provide recommendations on which tools, if any, may be suitable for adoption in new and developing EMS systems.

\section{Methods}

We used a scoping review methodology. A scoping review is "a way of mapping key concepts that underpin a research area" [11]. It is carried out to determine the extent of research available regarding a topic and is particularly useful in disciplines with emerging evidence [11].

We reviewed scientifically peer-reviewed published literature. We focused this review on triage tools used by EMS providers at the time of single patient care in the prehospital setting. Considering our focus on triage in routine EMS care, we did not include mass casualty triage tools given their unique mode of 
application to sorting patients in the specific circumstance of multicausality events. We defined prehospital triage as the algorithmic process undertaken by an EMS provider to sort the undifferentiated patient into an appropriate category based on suspected pathology and level of acuity. Clinical treatment protocols (e.g., step-by-step prehospital asthma treatment), clinical guidelines, and singular technologydependent triage tools (e.g., electrocardiogram (EKG) for prehospital STEMI triage) are excluded from our definition of triage.

A medical librarian performed a comprehensive literature search in December 2019. Relevant publications were identified by searching a combination of index terms and keywords for the concepts of triage and pre-hospital care in the following databases: MEDLINE (via Ovid MEDLINE® and Epub Ahead of Print, InProcess \& Other Non-Indexed Citations, Daily and Versions ${ }^{\circledR}, 1946$ to present), Embase (via Elsevier, Embase.com, 1947 to present), and Web of Science Core Collection (via Clarivate Analytics, including Science Citation Index Expanded 1974 to present, and Social Sciences Citation Index 1974 to present).

Results were limited to English language articles published between 2009 and 2019 to include more contemporaneous papers that are more likely to study tools in current use. Publication types were limited to the primary literature, including observational cohort and interventional studies. Since we sought to perform a direct review of the most robust primary literature studying these tools, we excluded case reports, reviews, systematic reviews, meta-analyses, comments, editorials, letters, and conference proceedings. All results were exported to, and deduplicated in, EndNote X9 (Clarivate Analytics, Philadelphia, PA). Covidence systematic review software (Veritas Health Innovation, Melbourne, Australia) was used for screening and full text review. See Additional file 1 for a list of all database search strategies.

Retrieved articles were independently screened by two trained reviewers (A1, A2), blinded to each other's reviews. During screening, each reviewer read article titles and abstracts to determine if they satisfied inclusion criteria, and to ensure they did not meet any exclusion criteria (see Table 1). Articles were scored as 'yes', 'no', or 'maybe'. Discrepant reviews, or any reviews marked as 'maybe', were adjudicated by a third reviewer (A3). 
Table 1

Article inclusion and exclusion criteria

\section{Inclusion Criteria}

Prehospital/EMS focused*

In-depth description of triage tool included ${ }^{\#}$

Triage tool/process must be a main focus $\$$

Traditional ground and aeromedical EMS system

Observational studies with $n \geq 50$

Interventional studies

\section{Exclusion Criteria}

In-hospital focus only

Hypothetical triage too!@

Systematic review/meta-analysis

Atypical EMS systems

Observational study with $\mathrm{n}<50$

Triage by EMS dispatch/communications center

Mass casualty triage tool

Termination of resuscitation tool

Prehospital clinical algorithm or protocol ${ }^{\wedge}$

* The study had to specifically include patient-level prehospital data.

\# The triage tool must be fully described within the article or through a provided reference. The tool should help the provider arrive at a specific, often binary, triage decision (e.g., Transport patient to trauma center or not; label patient as low or high acuity).

$\$$ Assessment of triage outcomes or process must be a stated primary or secondary objective of the study.

@ The triage tool is not actively used in prehospital clinical practice, is used for research purposes only, or is in development.

${ }^{\wedge}$ Excludes EMS agency prehospital algorithms or protocols used for clinical management en route (e.g., "asthma protocol") or those that rely on a single diagnostic tool such as a fingerstick glucose or EKG to make a triage decision (e.g., "chest pain protocol")

The final list of included ('yes') articles was divided between the two reviewers (A1, A2) for a full text review and critical synthesis. The full manuscript of each article was reviewed in detail, and if an article was deemed to meet one or more exclusion criteria, then it was excluded with reason(s) provided. Full text review articles were summarized in prose in a paragraph format (see Additional file 2) which note findings of most relevance to the research objectives. Data from articles were also coded in a summary table (see Additional file 3). The investigators independently appraised, then collectively discussed, all findings to reach consensus regarding key conclusions and recommendations.

The studies included in the final synthesis were assigned a four-tier quality rating (very low, low, moderate, or high) assessed by a customized scale based off the GRADE criteria, which included the study design, number of centers, and sample size (small < 300, moderate 300-1000, or large > 1000) [12]. For example, very low quality rating was assigned to retrospective observational studies that were single 
center or with small sample size, and a high quality rating was reserved for interventional, controlled, multi-center studies with large sample sizes.

\section{Results}

1521 unique articles were retrieved from database query (Fig. 1). After title and abstract screening, 72 (4.7\%) met inclusion criteria, and 1449 (95.3\%) were excluded. Out of 72 articles which had full-text reviews performed, 55 (3.6\% of 1521 unique articles) were deemed relevant and included in the full-text qualitative synthesis. 17 articles were excluded during full-text review with reasons cited in Fig. 1. The prose format synopsis of all 55 articles can be found in Additional file 2, and a summary table of each study can be found in Additional file 3 .

\section{Medical Conditions}

Of the 55 studies included in our final analysis, 19 (35\%) focused on stroke triage, 19 (35\%) on trauma triage, and $15(27 \%)$ on triage of general undifferentiated patients. Of the remaining 2 (3\%) studies, one addressed infectious disease triage [13] and the other addressed triage of patients with only nontraumatic chief complaints [14].

\section{Location and Design of Studies}

All studies were performed in World Bank designated high income countries, with 23 (42\%) in North America, 22 (40\%) in Europe, $6(11 \%)$ in East Asia and $4(7 \%)$ in Australia. All studies were prospective or retrospective observational cohort studies with the exception of one small randomized controlled clinical trial on stroke triage [15].

\section{Pediatric Populations}

Twelve studies within the general undifferentiated triage and trauma triage categories included the pediatric population, and four (7\%) focused on pediatric patients exclusively. These four studies examined the Rapid Emergency Triage and Treatment System-pediatrics (RETTS-p) tool used for general undifferentiated pediatric triage in Sweden [16, 17], the Centers for Disease Control and American College of Surgeons Committee on Trauma National Trauma Triage Protocol/Field Triage Decision Scheme (FTDS) for pediatric trauma in the United States [18], and regional pediatric trauma tools employed in England [19].

\section{General Undifferentiated Triage Tools}

The tools for general undifferentiated triage focused on standardized communication of level of acuity assignments between prehospital and emergency department providers. Frequently studied examples include the United Kingdom National Early Warning Score (NEWS), the Canadian Triage and Acuity Scale (CTAS) and the American Emergency Severity Index (ESI) [20, 21, 22]. Such tools incorporate prehospital vital signs and level of consciousness assessments [23, 24]; some also include chief complaint, exam 
findings, and anticipated resource needs as part of the algorithm [25, 26]. A wide variety of clinical end points were studied, including short-term, in-hospital and 30-day mortality, inter-rater reliability while using a singular scoring system (e.g., between dispatch, EMS provider, and emergency room triagist), correlation between differing prehospital and in-hospital scoring systems, need for lifesaving interventions within hours of ED arrival, and need for intensive care unit (ICU) admission. There is significant heterogeneity of clinical end points in the articles reporting all-comer triage tools. Consequently, a single triage tool in this group with the best performance metrics could not be identified.

\section{Stroke Triage}

From 19 (35\%) articles, we found 18 different stroke prehospital triage tools designed to aid with the recognition of acute stroke. The most commonly studied stroke triage tools were the Rapid Arterial Occlusion Evaluation (RACE, $n=5$ studies, 26\%), Cincinnati Prehospital Stroke Scale (CPSS; $n=4,21 \%$ ), Field Assessment for Stroke Triage for Emergency Destination (FAST-ED, $n=4,21 \%$ ), and Los Angeles Motor Scale (LAMS, $n=3,16 \%$ ). The authors stated the ultimate aim of prehospital stroke triage is to ensure timely transport of patients with acute ischemic stroke to designated stroke centers that have capabilities for neuroimaging, administration of thrombolytic agents and/or endovascular intervention. According to the authors, these tools also aim to channel patients presenting with stroke mimics, such as hypoglycemia or seizure, away from the major stroke centers to optimize health system resource utilization. Finally, several articles argued that stroke triage tools aim to be easy to use and efficient to administer in the prehospital setting, and to correlate well with the gold standard tools used by in-hospital providers, such as the National Institutes of Health stroke scale (NIHSS) [27]. Of the 19 articles assessed, $10(53 \%)$ used the NIHSS as the referential standard of comparison, or as a model from which scales were derived. Clinical end points for the stroke triage studies were diverse and included: detection of large vessel occlusion (LVO), diagnosis of stroke/transient ischemic attack, tissue plasminogen activator (tPA) administration rate, stroke team activation, accurate destination triage decision, and inter-rater reliability.

The highest quality studies for stroke triage evaluated the RACE scale. RACE evaluates five items: facial palsy, upper extremity paresis, lower extremity paresis, head and gaze deviation, and aphasia/agnosia, with a total score of 0 to 9. For example, in a large prospective study in Spain, Carrera et al. validated RACE among a cohort of 1822 patients and found a sensitivity of $84 \%$ and specificity of $60 \%$ for detecting large vessel occlusion (LVO) for RACE score $\geq 5.35 \%$ of the patients with a RACE $\geq 5$ had LVO, compared with $6 \%$ LVOs with a RACE $<5(p<0.001)$ [28]. Jumaa et al. found that RACE $\geq 5$ had a sensitivity of $77 \%$ and specificity of $75 \%$ for LVO eligible for mechanical thrombectomy among a cohort of 1147 patients in the United States [29]. Additional scales that have undergone head-to-head comparisons with RACE with comparable performance include the FAST-ED and the CPSS tools [30, 31]. The performance characteristics of FAST-ED and CPSS were assessed in $5(26 \%)$ of articles. Overall, both have comparable sensitivity (56-83\%) and specificity (60-89\%) for LVO prediction [30, 31].

\section{Trauma Triage - Ground EMS}


A common objective of included trauma triage articles was to accurately identify injured patients that require emergent transport to designated trauma centers. The studies on trauma triage more consistently used similar end points, including trauma center need, under-triage and over-triage rates, and survival to discharge. Trauma center need was uniformly defined as Injury Severity Score (ISS) $>15$, need for urgent surgical intervention, or need for intensive care unit level care.

The majority of trauma triage tools identified are based off of the FTDS [32] which appears to be the de facto standard in studies originating from the USA. Since its initial publication in 1986, the FTDS has been revised five times: in 1990, 1993, 1999, 2006 and 2011. According to the articles, the FTDS uses stepwise identification of four aspects of clinical presentation involving physiologic criteria, anatomic criteria, mechanism of injury criteria, and special considerations criteria to identify patients requiring transport to a trauma center. Physiologic criteria focus on vital signs and Glasgow Coma Scale (GCS); anatomic criteria include specific severe injury patterns such as penetrating trauma, flail chest and crush injury; mechanism of injury criteria focuses on high energy mechanisms such as falls from specific height, high speed vehicular crash, and motorcycle accidents; and special considerations include extremes of age, high risk comorbidities, burns, pregnancy, and anticoagulated status [32].

The highest quality study of FTDS performance within this scoping review was conducted by Newgard et al. in 2011; it evaluated the performance characteristics of the 2006 version of FTDS, with a cohort of 122,345 injured patients evaluated and transported by EMS over a 3-year period [33]. Major trauma was defined as ISS $>15$, and the overall sensitivity and specificity of the FTDS criteria for identifying major trauma patients were $86 \%(95 \% \mathrm{Cl} 85-87 \%)$ and $69 \%$ (95\% $\mathrm{Cl} 68-69 \%)$, respectively. Triage sensitivity and specificity, respectively, differed by age: $84 \%$ and $66 \%$ ( 0 to 17 years); $90 \%$ and $64 \%$ (18 to 54 years); and $80 \%$ and $75 \%$ ( $\geq 55$ years). Overall, FTDS appears to have comparatively reduced sensitivity and increased specificity in detection of trauma in elderly patients.

Other frequently studied ground EMS tools included the Vittel criteria (France) [34, 35], Dutch Field Triage Protocol (Netherlands) [36, 37], and Prehospital Index (Canada) [37, 38].

\section{Trauma Triage - Aeromedical EMS}

Three studies focused on the use of trauma triage tools to decide on the utility of helicopter transport [39, $40,41]$. Brown et al. conducted a US retrospective cohort study of 258,387 trauma patients $(16 \%$ transported by helicopter, remainder by ground) and found odds of increased survival to discharge for patients transported by helicopter in the following FTDS conditions: GCS < 14 (adjusted Odds Ratio 1.22); respiratory rate $<10$ or $>29$ (aOR 1.32), penetrating injury (aOR 1.40), or age $>55$ (aoR 1.15) [40]. In 2017, Brown et al. investigated the Air Medical Prehospital Triage (AMPT) score, which awards points for low GCS, abnormal respiratory rate, unstable chest wall injury patterns, paralysis, multisystem trauma, or fulfillment of any physiologic plus anatomic criterion from FTDS. The authors found that helicopter EMS increases odds of in-hospital survival by $6.7 \%$ for patients with AMPT score $\geq 2$ (Absolute Risk Reduction $1.067 ; 95 \% \mathrm{Cl} 1.040-1.083, p<0.001, n=222,827)[41]$.

\section{Trauma Triage - Traumatic Brain Injury}


Two studies by Fuller et al. focused on predictive tools for triaging severe traumatic brain injury (TBI) in the field $[42,43]$. The authors studied the Head Injury Transportation Straight to Neurosurgery study (HITS-NS) triage tool and London Ambulance Service major trauma triage tool and found that both had poor sensitivity $(<45 \%)$ for detection of severe TBI which was concerning for EMS providers missing TBls $[42,43]$.

\section{Simplifying Triage Tools}

While most trauma triage studies investigated performance characteristics of established tools, a subset attempted to identify ways to further simplify tools for EMS providers [34,44]. These studies emphasized the challenges of designing the ideal triage tool: the design must optimize over and under-triage rates while remaining streamlined and user friendly to promote widespread adoption.

\section{Discussion}

Our scoping review found 55 studies on prehospital triage tools published within the past decade. These tools focused on general undifferentiated, trauma, and stroke populations and all included studies originated from high-income countries. Studies predominantly sought to assess predictive accuracy of the triage tools compared to in-hospital clinical outcomes, and many studied accuracy in simplified versions of existing tools. These published triage tools are generally designed to help prehospital providers determine destination of transport, means of transport and level of acuity. These tools also appear to provide a shared language for prehospital personnel to communicate with other emergency personnel, and assist in identifying vital sign derangements and exam findings across a spectrum of age ranges to differentiate 'acute' and 'non-acute' patients.

Trauma and stroke tools comprised over two-thirds of the included articles, perhaps because of their clinical and health systems significance. Outcomes for trauma and stroke depend on timely field recognition and are influenced by highly time sensitive interventions that are destination-dependent [45, 46]. Further, trauma and stroke care are regionalized in many high-income countries, therefore right patient destination are important to study for trauma and stroke system optimization. Last, both stroke and trauma outcomes are used to drive 'benchmarking' for health system accreditation and funding, which may also drive their importance as a research topic $[47,48,49]$.

In trauma, the US FTDS appears to be the "industry standard" triage tool used, likely reflecting that the majority of our studies were from North America, specifically, the USA. As the majority of tools within the trauma triage literature derive from the FTDS, this well-researched tool is a promising starting point for further simplified trauma triage tool development, such as identifying individual components that may predict clinically relevant trauma outcomes. The trauma literature was relatively cohesive in that most studies used common clinical end points, which facilitates comparisons across studies.

In stroke care, while no single tool emerged as the prehospital triage 'gold' standard, the RACE, FAST-ED and Cincinnati Prehospital Stroke scales appear to have the highest quality data supporting their use. The 
National Institutes of Health Stroke Scale was presented in multiple studies as the gold standard inhospital tool which was used for comparison.

The all-comer triage literature includes a myriad of tools with varying complexity, from those that incorporate vital signs alone (e.g., NEWS), to those with complex diagnostic algorithms incorporating history and exam findings to arrive at a level of acuity designation (e.g., CTAS). No one tool emerged as a clear gold standard, and authors' use of a wide variety of clinical end points which make cross comparisons challenging.

Research themes common to these studies include simplifying existing tools such that they are efficient and accurate for the EMS provider to derive an accurate triage decision, and to identify the most accurate tool out of a large cadre of tools currently available. Standardized reporting of clinical end points would facilitate this endeavor in future research. Additionally, we noted a paucity of articles researching implementation or assessing end user perspectives [28, 50,51], and no studies examined costs associated with triage decisions. Qualitative studies, cost analyses, and implementation studies would be helpful to further our understanding of the value provided by prehospital triage tools.

Lastly, all the studies included in this scoping review were performed in a few high-income settings, and the tools may not translate well to other high-income settings or LMICs with a different healthcare configuration, infrastructure and cadres of prehospital providers. Destination decision making would need to be locally-determined, especially in LMICs where specialty diagnostic (e.g., computed tomography scanners) and therapeutic resources (e.g., tPA) may be even more scarce. Further, triage tools may need to be tailored based upon regional injury and illness patterns. For example, prehospital triage of obstetric emergencies was notably missing from our review. Jenson et al. performed a systematic review of emergency department (i.e. in hospital) triage tools in LMICs and identified the South Africa Triage Scale (SATS), modified Early Warning Score and the Australasian Triage Scale as promising tools that had been validated across multiple studies in LMIC settings [8]. SATS has been implemented in the prehospital setting in South Africa and studies analyzing performance characteristics, while on-going, are yet to be published [52].

In recent years, prehospital care has received increased recognition in international health policy. Data extrapolated from the Global Burden of Disease study show that 24 million lives are lost each year in LMICs due to conditions sensitive to prehospital and emergency care. Ischemic heart disease, cerebrovascular accidents, and unintentional injuries are the largest contributors to morbidity and mortality in these settings [6, 53]. In 2019, delegates to the 72nd World Health Assembly adopted a resolution to strengthen emergency and trauma care systems and prehospital care was highlighted as an essential component [54]. Prehospital triage tools are a key building block for quality and safety assurance in the development of novel EMS systems [55]. It is our hope that this scoping review has provided a valuable framework for what is known thus far, and that further research will be done to advance the field. 
The authors acknowledge the following limitations of this scoping review. First, the review was limited to English language publications. This may have excluded triage tools published in non-English journals. Secondly, the review was limited to only peer-reviewed published literature; it is likely that white papers and other non-peer-reviewed papers discuss additional triage tools currently in use. Lastly, inherent to this study's design as a scoping review, the authors were unable to draw quantitative conclusions about the performance characteristics of the tools presented.

\section{Conclusions}

This scoping review found that the majority of literature on prehospital triage focused on trauma and stroke specifically, with a few reports on triage tools for general undifferentiated patients. Much of this body of work originates from high income countries. The Field Triage Decision Scheme for trauma, and the Rapid Arterial Occlusion Evaluation for stroke, are especially well studied tools which may serve as tools for emerging EMS systems or as good starting points for simplified adaptations for established EMS systems. We found no single universally accepted 'standard' prehospital triage tool. Future research should focus on implementation analysis and real-world application of these tools. Additionally, research efforts should focus on the development a single universal triage tool that can be adapted for a variety of contexts.

\section{Abbreviations}

AMPT

Air Medical Prehospital Triage

$\mathrm{aOR}$

adjusted odds ratio

CPSS

Cincinnati Prehospital Stroke Scale

CTAS

Canadian Triage and Acuity Scale

EKG

electrocardiogram

EMS

Emergency medical services

ESI

Emergency Severity Index

FAST-ED

Field Assessment for Stroke Triage for Emergency Destination

FTDS

Field Triage Decision Scheme

GCS 
Glasgow Coma Scale

HITS-NS

Head Injury Transportation Straight to Neurosurgery

ICU

Intensive care unit

LAMS

Los Angeles Motor Scale

LMICs

Low- and middle-income countries

LVO

Large vessel occlusion

NEWS

National Early Warning Score

NIHSS

National Institutes of Health Stroke Scale

RACE

Rapid Arterial Occlusion Evaluation

RETTS-p

Rapid Emergency Triage and Treatment System - pediatrics

SATS

South Africa Triage Scale

STEMI

ST elevation myocardial infarction

TBI

traumatic brain injury

tPA

tissue plasminogen activator

\section{Declarations}

\section{Ethics approval and consent to participate}

Not applicable as this study is classified as non-human subjects research by the Colorado Multiple Institutional Review Board (Human Research Protection Program Policies \& Procedures, September 2011).

\section{Consent for publication}

Not applicable.

\section{Availability of data and materials}


All data generated or analyzed during this study are included in this published article [and its supplementary information files].

\section{Competing interests}

The authors declare that they have no competing interests

\section{Funding}

There was no source of funding for this project.

\section{Authors' contributions}

NM conceptualized this study and outlined the manuscript; KS performed the database query; SB and MH performed the title/abstract screening and full text reviews and NM adjudicated discrepant reviews; SB and NM drafted the manuscript; LAW, MH, NM and CD provided critical revisions to the manuscript; LAW and WB provided content expertise; all authors read and approved the final manuscript.

\section{Acknowledgements}

The authors thank Ms. Chandni Patel for her editorial assistance.

\section{References}

1. Moore L. Measuring quality and effectiveness of prehospital EMS. Prehosp Emerg Care. 1999;3(4):325-31.

2. Mahmoud KD, Gu YL, Nijsten MW, de Vos R, Nieuwland W, Zijlstra F, et al. Interhospital transfer due to failed prehospital diagnosis for primary percutaneous coronary intervention: an observational study on incidence, predictors, and clinical impact:. European Heart Journal: Acute Cardiovascular Care. 2013;2(2):166-75.

3. Xian Y, Holloway RG, Chan PS, Noyes K, Shah MN, Ting HH, et al. Association between stroke center hospitalization for acute ischemic stroke and mortality. JAMA. 2011;305(4):373-80.

4. Haas B, Stukel TA, Gomez D, Zagorski B, De Mestral C, Sharma SV, et al. The mortality benefit of direct trauma center transport in a regional trauma system: a population-based analysis. J Trauma Acute Care Surg. 2012;72(6):1510-5-discussion1515-7.

5. Robertson-Steel I. Evolution of triage systems. Emerg Med J. 2006;23(2):154-5.

6. Hsia RY, Thind A, Zakariah A, Hicks ER, Mock C. Prehospital and Emergency Care: Updates from the Disease Control Priorities, Version 3. World J Surg. 2nd ed. 2015;39(9):2161-7.

7. Suryanto, Plummer V, Boyle M. EMS Systems in Lower-Middle Income Countries: A Literature Review. Prehosp Disaster Med. 2017;32(1):64-70.

8. Jenson A, Hansoti B, Rothman R, de Ramirez SS, Lobner K, Wallis L. Reliability and validity of emergency department triage tools in low- and middle-income countries: a systematic review. 
European Journal of Emergency Medicine. 2018;25(3):154-60.

9. Mould-Millman N-K, Dixon JM, Sefa N, Yancey A, Hollong BG, Hagahmed M, et al. The State of Emergency Medical Services (EMS) Systems in Africa. Prehosp Disaster Med. 2017;32(3):273-83.

10. Lidal IB, Holte HH, Vist GE. Triage systems for pre-hospital emergency medical services - a systematic review. Scand J Trauma Resusc Emerg Med. 2013;21(1):28-6.

11. Peters MDJ, Godfrey CM, Khalil H, Mclnerney P, Parker D, Soares CB. Guidance for conducting systematic scoping reviews. Int J Evid Based Healthc. 2015;13(3):141-6.

12. Guyatt GH, Oxman AD, Vist GE, Kunz R, Falck-Ytter Y, Alonso-Coello P, et al. GRADE: an emerging consensus on rating quality of evidence and strength of recommendations. BMJ. 2008;336(7650):924-6.

13. Johansson N, Spindler C, Valik J, Vicente V. Developing a decision support system for patients with severe infection conditions in pre-hospital care. Int J Infect Dis. 2018;72:40-8.

14. Gaumont D, Cummins N, Hannigan A, Ryan D. ViEWS from the prehospital perspective: a comparison with a prehospital score to triage categorisation in the emergency department. Ir Med J. 2016;109(6):423.

15. Helwig SA, Ragoschke-Schumm A, Schwindling L, Kettner M, Roumia S, Kulikovski J, et al. Prehospital Stroke Management Optimized by Use of Clinical Scoring vs Mobile Stroke Unit for Triage of Patients With Stroke: A Randomized Clinical Trial. JAMA Neurol. 2019;76(12):1484-92.

16. Magnusson C, Herlitz J, Karlsson T, Axelsson C. Initial assessment, level of care and outcome among children who were seen by emergency medical services: a prospective observational study. Scand J Trauma Resusc Emerg Med. 2018;26(1):88-11.

17. Magnusson C, Herlitz J, Karlsson T, Jiménez-Herrera M, Axelsson C. The performance of the EMS triage (RETTS-p) and the agreement between the field assessment and final hospital diagnosis: a prospective observational study among children < 16 years. BMC Pediatr. 2019;19(1):500-10.

18. Lerner EB, Cushman JT, Drendel AL, Badawy M, Shah MN, Guse CE, et al. Effect of the 2011 Revisions to the Field Triage Guidelines on Under- and Over-Triage Rates for Pediatric Trauma Patients. Prehosp Emerg Care. 2017;21(4):456-60.

19. Ardolino A, Cheung CR, Lawrence T, Bouamra O, Lecky F, Berry K, et al. The accuracy of existing prehospital triage tools for injured children in England: an analysis using emergency department data. Emerg Med J. 2015;32(5):397-400.

20. McGinley A, Pearse RM. A national early warning score for acutely ill patients. BMJ. 2012;345(aug08 1):e5310-0.

21. J Murray M. The Canadian Triage and Acuity Scale: A Canadian perspective on emergency department triage. Emerg Med. 2003;15(1):6-10.

22. Wuerz RC, Milne LW, Eitel DR, Travers D, Gilboy N. Reliability and validity of a new five-level triage instrument. Acad Emerg Med. 2000;7(3):236-42. 
23. Hoikka M, Silfvast T, Ala-Kokko TI. Does the prehospital National Early Warning Score predict the short-term mortality of unselected emergency patients? Scand J Trauma Resusc Emerg Med. 2018;26(1):48-7.

24. Leung SC, Leung LP, Fan KL, Yip WL. Can prehospital Modified Early Warning Score identify nontrauma patients requiring life-saving intervention in the emergency department? Emerg Med Australas. 2016;28(1):84-9.

25. Buschhorn HM, Strout TD, Sholl JM, Baumann MR. Emergency medical services triage using the emergency severity index: is it reliable and valid? J Emerg Nurs. 2013;39(5):e55-63.

26. Leeies M, Ffrench C, Strome T, Weldon E, Bullard M, Grierson R. Prehospital Application of the Canadian Triage and Acuity Scale by Emergency Medical Services. CJEM. 2017;19(1):26-31.

27. Lyden P. Using the National Institutes of Health Stroke Scale: A Cautionary Tale. Stroke. 2017;48(2):513-9.

28. Carrera D, Gorchs M, Querol M, Abilleira S, Ribó M, Millán M, et al. Revalidation of the RACE scale after its regional implementation in Catalonia: a triage tool for large vessel occlusion. J Neurointerv Surg. 2019;11(8):751-6.

29. Jumaa MA, Castonguay AC, Salahuddin H, Shawver J, Saju L, Burgess R, et al. Long-term implementation of a prehospital severity scale for EMS triage of acute stroke: a real-world experience. Journal of Neurointerventional Surgery. 2019;02:02.

30. Lima FO, Silva GS, Furie KL, Frankel MR, Lev MH, Camargo ÉCS, et al. Field Assessment Stroke Triage for Emergency Destination: A Simple and Accurate Prehospital Scale to Detect Large Vessel Occlusion Strokes. Stroke. 2016;47(8):1997-2002.

31. Anadani M, Almallouhi E, Wahlquist AE, Debenham E, Holmstedt CA. The Accuracy of Large Vessel Occlusion Recognition Scales in Telestroke Setting. Telemed J E Health. 2019;25(11):1071-6.

32. Sasser SM, Hunt RC, Faul M, and DSR, 2012. Guidelines for field triage of injured patients: recommendations of the National Expert Panel on Field Triage, 2011. MMWR Recomm Rep. 2012;61(RR-1):1-20.

33. Newgard CD, Zive D, Holmes JF, Bulger EM, Staudenmayer K, Liao M, et al. A multisite assessment of the American College of Surgeons Committee on Trauma field triage decision scheme for identifying seriously injured children and adults. J Am Coll Surg. 2011;213(6):709-21.

34. Cassignol A, Marmin J, Cotte J, Cardinale M, Bordes J, Pauly V, et al. Correlation between field triage criteria and the injury severity score of trauma patients in a French inclusive regional trauma system. Scand J Trauma Resusc Emerg Med. 2019;27(1):71-9.

35. Cassignol A, Markarian T, Cotte J, Marmin J, Nguyen C, Cardinale M, et al. Evaluation and Comparison of Different Prehospital Triage Scores of Trauma Patients on In-Hospital Mortality. Prehosp Emerg Care. 2019;23(4):543-50.

36. van Laarhoven JJEM, Lansink KWW, van Heijl M, Lichtveld RA, Leenen LPH. Accuracy of the field triage protocol in selecting severely injured patients after high energy trauma. Injury. 2014;45(5):869-73. 
37. Voskens FJ, van Rein EAJ, van der Sluijs R, Houwert RM, Lichtveld RA, Verleisdonk EJ, et al. Accuracy of Prehospital Triage in Selecting Severely Injured Trauma Patients. JAMA Surg. 2018;153(4):322-7.

38. Lavoie A, Emond M, Moore L, Camden S, Liberman M. Evaluation of the Prehospital Index, presence of high-velocity impact and judgment of emergency medical technicians as criteria for trauma triage. CJEM. 2010;12(2):111-8.

39. Giannakopoulos GF, Saltzherr TP, Lubbers WD, Christiaans HMT, van Exter P, de Lange-de Klerk ESM, et al. Is a maximum Revised Trauma Score a safe triage tool for Helicopter Emergency Medical Services cancellations? Eur J Emerg Med. 2011;18(4):197-201.

40. Brown JB, Forsythe RM, Stassen NA, Gestring ML. The National Trauma Triage Protocol: can this tool predict which patients with trauma will benefit from helicopter transport? J Trauma Acute Care Surg. 2012;73(2):319-25.

41. Brown JB, Gestring ML, Guyette FX, Rosengart MR, Stassen NA, Forsythe RM, et al. External validation of the Air Medical Prehospital Triage score for identifying trauma patients likely to benefit from scene helicopter transport. J Trauma Acute Care Surg. 2017;82(2):270-9.

42. Fuller G, Lawrence $T$, Woodford M, Lecky F. The accuracy of alternative triage rules for identification of significant traumatic brain injury: a diagnostic cohort study. Emergency Medicine Journal. 2014;31(11):914-9.

43. Fuller G, McClelland G, Lawrence T, Russell W, Lecky F. The diagnostic accuracy of the HITSNS prehospital triage rule for identifying patients with significant traumatic brain injury: a cohort study. European Journal of Emergency Medicine. 2016;23(1):61-4.

44. Brown JB, Stassen NA, Bankey PE, Sangosanya AT, Cheng JD, Gestring ML. Mechanism of injury and special consideration criteria still matter: an evaluation of the National Trauma Triage Protocol. J Trauma. 2011;70(1):38-44-discussion44-5.

45. Nathens AB, Jurkovich GJ, Cummings P, Rivara FP, Maier RV. The Effect of Organized Systems of Trauma Care on Motor Vehicle Crash Mortality. JAMA. 2000;283(15):1990-4.

46. Fassbender K, Balucani C, Walter S, Levine SR, Haass A, Grotta J. Streamlining of prehospital stroke management: the golden hour. The Lancet Neurology. 2013;12(6):585-96.

47. Lichtman JH, Jones SB, Wang Y, Watanabe E, Leifheit-Limson E, Goldstein LB. Outcomes after ischemic stroke for hospitals with and without Joint Commission-certified primary stroke centers. Neurology. 2011;76(23):1976-82.

48. MacKenzie EJ, Rivara FP, Jurkovich GJ, Nathens AB, Frey KP, Egleston BL, et al. A national evaluation of the effect of trauma-center care on mortality. N Engl J Med. 2006;354(4):366-78.

49. Batomen B, Moore L, Strumpf E, Champion H, Nandi A. Impact of trauma center accreditation on mortality and complications in a Canadian trauma system: an interrupted time series analysis. BMJ Qual Saf. 2020.

50. Carr K, Yang Y, Roach A, Shivashankar R, Pasquale D, Serulle Y. Mechanical Revascularization in the Era of the Field Assessment Stroke Triage for Emergency Destination (FAST-ED): A Retrospective Cohort Assessment in a Community Stroke Practice. J Stroke Cerebrovasc Dis. 2020;29(1):104472. 
51. Barnett AS, Wang NE, Sahni R, Hsia RY, Haukoos JS, Barton ED, et al. Variation in prehospital use and uptake of the national Field Triage Decision Scheme. Prehosp Emerg Care. 2013;17(2):135-48.

52. Mould-Millman NK, Colborn K, De Vries S, Dixon J, Hodkinson P, Ginde A, et al. 163 Out-of-Hospital Application of the South African Triage Scale (SATS): Discordance Between Gold Standard and Medic Triage. Annals of Emergency Medicine. 2016;4(S)(68):S64.

53. Lozano R, Naghavi M, Foreman K, Lim S, Shibuya K, Aboyans V, et al. Global and regional mortality from 235 causes of death for 20 age groups in 1990 and 2010: a systematic analysis for the Global Burden of Disease Study 2010. Lancet. 2012;380(9859):2095-128.

54. World Health Organization. 72nd World Health Assembly adopts resolution on emergency and trauma care. https://www.who.int/news/item/27-05-2019-72nd-world-health-assembly-adoptsresolution-on-emergency-and-trauma-care. Accessed 10 January 2021.

55. Mehmood A, Rowther AA, Kobusingye O, Hyder AA. Assessment of pre-hospital emergency medical services in low-income settings using a health systems approach. Int J Emerg Med. 2018;11(1):5310.

\section{Figures}




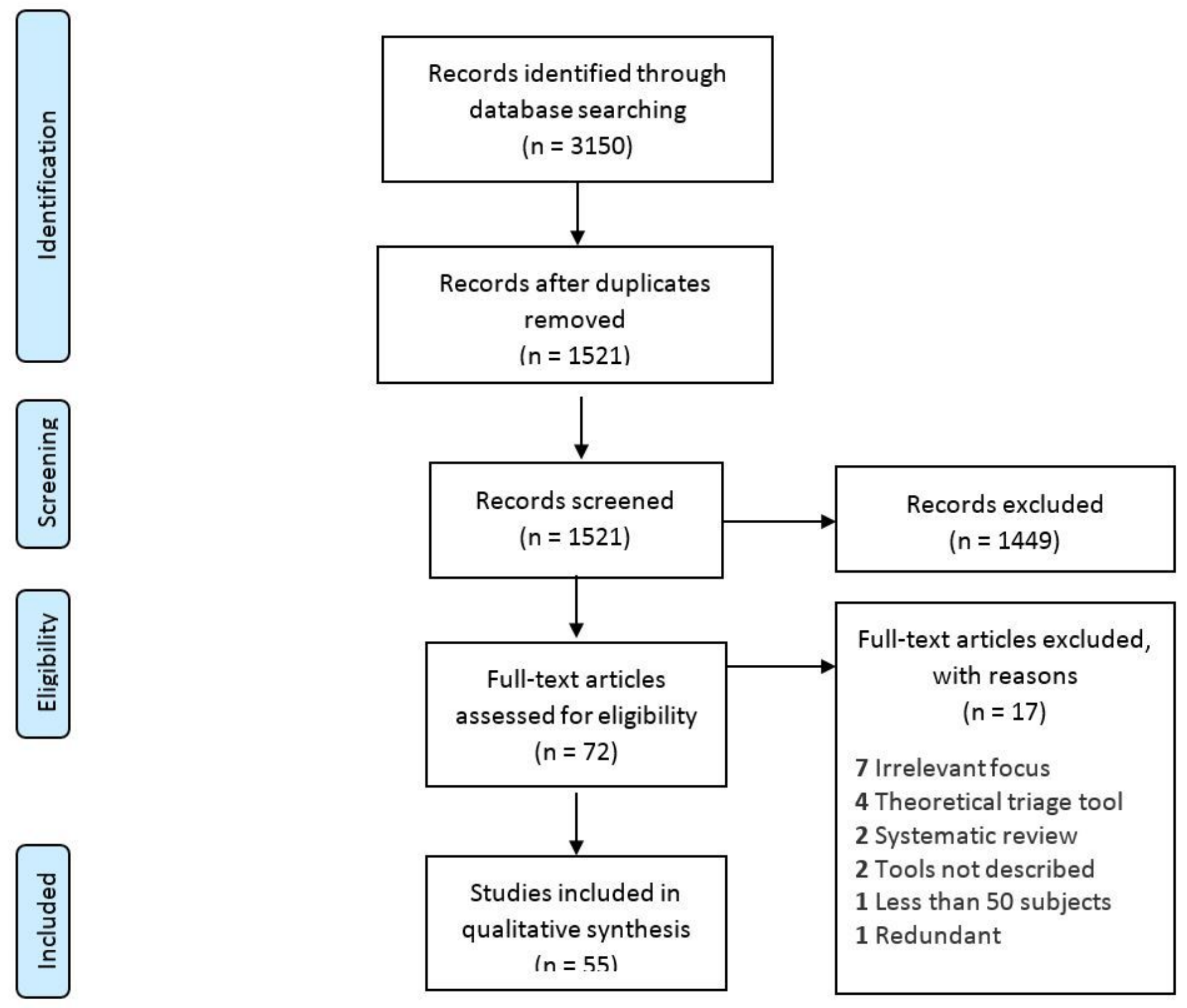

Figure 1

PRISMA flow diagram of articles.

\section{Supplementary Files}

This is a list of supplementary files associated with this preprint. Click to download.

- Additionalfile115JAN2021.docx

- Additionalfile215JAN2021.docx

- Additionalfile315JAN2021.xlsx 